\title{
A martingale approach to general Franklin systems
}

\author{
by \\ Anna Kamont (Sopot) and Paul F. X. Müller (Linz)
}

\begin{abstract}
We prove unconditionality of general Franklin systems in $L^{p}(X)$, where $X$ is a UMD space and where the general Franklin system corresponds to a quasi-dyadic, weakly regular sequence of knots.
\end{abstract}

\section{INTRODUCTION}

In this paper we continue the line of research that extends properties of biorthogonal systems from the case of scalar-valued function spaces to their UMD-valued analogues. We are particularly concerned with the Franklin system and its generalizations corresponding to non-dyadic partitions. It is well known that the classical Franklin system is a basis in $L^{p}[0,1]$, $1 \leq p<\infty$, and in $C[0,1]$, and it has been proved by S. V. Bochkarev [1] that this basis is unconditional when $1<p<\infty$. Moreover, Z. Ciesielski, P. Simon and P. Sjölin [5] have proved that the Franklin and Haar systems are equivalent in $L^{p}[0,1]$ for $1<p<\infty$.

In the vector-valued case, the problem of unconditionality of the Franklin system in $L^{p}(X)$ has been solved for UMD spaces by T. Figiel, who developed a general martingale approach to such problems in [6] and [7]. Summaries of Figiel's work appear in [8] and [13].

A general Franklin system on $[0,1]$ consists of piecewise linear functions corresponding to an arbitrary sequence of knots, dense in $[0,1]$. By the work of Z. Ciesielski [4] from 1963, general Franklin systems are known to be bases in $L^{p}[0,1], 1 \leq p<\infty$, and in $C[0,1]$ (the latter when all knots are simple, i.e. all functions from the system in question are continuous). By contrast, the problem of unconditionality for general Franklin systems has not been

2000 Mathematics Subject Classification: 42C15, 46E40, 60G46.

Key words and phrases: general Franklin system, vector-valued $L^{p}$ space, UMD space, unconditionality, martingale.

A. Kamont was supported by FWF project P150907-N08 and KBN grant 1 P03A 038 27; she also gratefully acknowledges the support of the Foundation for Polish Science.

P. Müller was supported by FWF project P150907-N08. 
studied until a series of recent papers [10], [12], [11]. The final answer to this problem is in [11]: for an arbitrary sequence of knots, the corresponding general Franklin system is an unconditional basis in $L^{p}[0,1], 1<p<\infty$.

In this paper, we are interested in the question of unconditionality of general Franklin systems in $L^{p}(X)=L^{p}([0,1], X)$, where $X$ is a UMD space. We answer this question in the affirmative when the system corresponds to a quasi-dyadic, weakly regular sequence of knots. The method of proof is an extension of T. Figiel's martingale approach. In this paper we exploit J. Bourgain's version of E. M. Stein's martingale inequality to estimate the martingales resulting from Figiel's expansion of Franklin functions. Thus Stein's inequality replaces the use of explicit martingale transforms appearing in Figiel's original work [6].

\section{THE MAIN RESULT}

In this section we state the main result of this paper concerning $L^{p}(X)$ equivalence between the general Haar system and the general Franklin system built on quasi-dyadic sequences of partitions. We first review these notions and recall the relevant definitions.

Quasi-dyadic sequences of partitions. Let $\left\{\mathcal{P}_{j}, j \geq 0\right\}$ be a quasi-dyadic sequence of partitions of $[0,1]$. This means that $\mathcal{P}_{j}=\left\{t_{j, k}, 0 \leq k \leq 2^{j}\right\}$ with $0=t_{j, 0}<t_{j, 1}<\cdots<t_{j, 2^{j}-1}<t_{j, 2^{j}}=1$ and $t_{j, k}=t_{j+1,2 k}, \quad 0 \leq k \leq 2^{j}$. That is, $\mathcal{P}_{j+1}$ is obtained from $\mathcal{P}_{j}$ by inserting a new point between any two points of $\mathcal{P}_{j}$. The following notation will be used:

$$
\begin{aligned}
I_{j, k} & =\left[t_{j, k-1}, t_{j, k}\right], \quad 1 \leq k \leq 2^{j}, \\
\mathcal{I}_{j} & =\left\{I_{j, k}, 1 \leq k \leq 2^{j}\right\}, \quad \mathcal{I}=\bigcup_{j \geq 0} \mathcal{I}_{j} .
\end{aligned}
$$

For $I=I_{j, k}$ we write $I^{-}=I_{j, k-1}, I^{+}=I_{j, k+1}$. Moreover, we enumerate the intervals $\mathcal{I}$ by putting

$$
I_{2^{j}+k}=I_{j, k}, \quad j \geq 0,1 \leq k \leq 2^{j} .
$$

General Franklin system corresponding to a quasi-dyadic sequence of partitions. Let $\left\{\mathcal{P}_{j}, j \geq 0\right\}$ be a quasi-dyadic sequence of partitions. Let $\pi_{1}=\{0,1\}, t_{0}=0, t_{1}=1$, and for $n \geq 2, n=2^{j}+k$ put $t_{n}=t_{j+1,2 k-1}$, $\pi_{n}=\left\{t_{0}, \ldots, t_{n}\right\}$; more precisely, $\pi_{n}$ is the partition of $[0,1]$ obtained by increasing rearrangement of the points $t_{0}, \ldots, t_{n}$, and the point $t_{n}=t_{j+1,2 k-1}$ is inserted into the interval $I_{j, k}$. We denote by $S_{n}$ the space of continuous and piecewise linear functions on $[0,1]$ with knots $\pi_{n}$. Since $\operatorname{dim} S_{n}=$ $\operatorname{dim} S_{n-1}+1$, for each $n \geq 2$ there is a unique function $f_{n} \in S_{n}$ such that $f_{n}$ is orthogonal (in $L^{2}[0,1]$ ) to $S_{n-1},\left\|f_{n}\right\|_{2}=1$ and $f_{n}\left(t_{n}\right)>0$. Letting $f_{0} \equiv 1$ and $f_{1}(t)=\sqrt{3}(2 t-1)$, we obtain a complete orthonormal system 
$\left\{f_{n}, n \geq 0\right\}$, which is called the general Franklin system corresponding to the quasi-dyadic sequence of partitions $\left\{\mathcal{P}_{j}, j \geq 0\right\}$.

For convenience, we set $\phi_{n}=f_{n} /\left\|f_{n}\right\|_{\infty}$, and we write $\phi_{n}=\phi_{j, k}$, where $n=2^{j}+k$ with $j \geq 0$ and $1 \leq k \leq 2^{j}$. We also use the abbreviation $1_{j, k}=1_{I_{j, k}}$.

General Haar system corresponding to a quasi-dyadic sequence of partitions. We will also need the general Haar system corresponding to $\left\{\mathcal{P}_{j}\right.$, $j \geq 0\}$. For $I \in \mathcal{I}, I=I_{j, k}$, denote by $h_{I}$ or $h_{j, k}$ the $L^{\infty}$ normalized Haar function, supported on $I=I_{j, k}$, that corresponds to the splitting $I_{j, k}=I_{j+1,2 k-1} \cup I_{j+1,2 k}$. Explicitly $h_{I_{j, k}}$ is given as

$$
h_{I_{j, k}}=\frac{\left|I_{j+1,2 k-1}\right| \wedge\left|I_{j+1,2 k}\right|}{\left|I_{j+1,2 k-1}\right|} 1_{I_{j+1,2 k-1}}-\frac{\left|I_{j+1,2 k-1}\right| \wedge\left|I_{j+1,2 k}\right|}{\left|I_{j+1,2 k}\right|} 1_{I_{j+1,2 k}},
$$

where $1_{A}$ is the indicator function of the set $A$. In what follows, we use the abbreviations $h_{I_{j, k}}=h_{j, k}=h_{n}$ with $n=2^{j}+k$.

Comment. The above extends the purely dyadic case: if we specialize $t_{j, k}=k / 2^{j}$, then $I_{j, k}$ defined by (2.1) is just a dyadic interval, and $h_{I_{j, k}}$ defined by (2.4) is the $L^{\infty}$ normalized dyadic Haar function.

A dyadic Haar function is distinguished by the fact that its absolute value is constant (and equal to 1) on its support. Next we impose a well known regularity condition on the quasi-dyadic partition ensuring that the moduli of the Haar functions defined by (2.4) satisfy uniform lower bounds on their supports. This regularity condition arose in the study of martingale inequalities associated to increasing sequences of $\sigma$-algebras through the work of R. Gundy and D. L. Burkholder in the 1960's (see for example A. M. Garsia's book [9]). Following the terminology used by approximation theorists, we call this property the weak regularity condition.

Weak regularity condition for a quasi-dyadic sequence of partitions. For our main result, we assume the following regularity condition (weak regularity condition) for the sequence of partitions $\left\{\mathcal{P}_{j}, j \geq 0\right\}$ : there is a parameter $\gamma \geq 1$ such that

$$
\frac{1}{\gamma} \leq \frac{\left|I_{j+1,2 k-1}\right|}{\left|I_{j+1,2 k}\right|} \leq \gamma \quad \text { for all } j \geq 0,1 \leq k \leq 2^{j} .
$$

This condition means that when $I_{j, k}$ is split by the point $t_{j+1,2 k-1}$ into $I_{j+1,2 k-1} \cup I_{j+1,2 k}$, then the lengths of $I_{j+1,2 k-1}$ and $I_{j+1,2 k}$ are comparable. In particular, the weak regularity condition with parameter $\gamma$ implies that

$$
\frac{1}{\gamma+1}\left|I_{j, k}\right| \leq\left|I_{j+1,2 k-1}\right|,\left|I_{j+1,2 k}\right| \leq \frac{\gamma}{\gamma+1}\left|I_{j, k}\right| .
$$

The above definitions extend naturally the notion of dyadic intervals, the dyadic Haar system and the classical Franklin system. The main results 
relating the Franklin system and the Haar system assert that the classical Franklin system is an unconditional basis in $L^{p}[0,1], 1<p<\infty$ (Theorem of S. V. Bochkarev [1]), and that furthermore the Franklin and Haar systems form equivalent bases in the reflexive $L^{p}[0,1]$ spaces (Theorem of Z. Ciesielski, P. Simon and P. Sjölin [5]). Extending the classical results, T. Figiel [6] showed that for any UMD space $X$ the Franklin and Haar systems are equivalent in the vector-valued $L^{p}$ space $L^{p}(X)$. Since the classical work relied on tools that are strictly limited to the scalar case, T. Figiel had to invent a new - martingale-based - approach to biorthogonal systems.

In a different direction, the results of S. V. Bochkarev and Z. Ciesielski, P. Simon and P. Sjölin were extended to the case of general Franklin systems: For quasi-dyadic partitions, and under the condition of weak regularity, it was proved in [10] that unconditionality holds for $\left\{\phi_{n}\right\}_{n=0}^{\infty}$ in the reflexive $L^{p}[0,1]$ spaces and moreover that $\left\{h_{n}\right\}_{n=1}^{\infty}$ and $\left\{\phi_{n}\right\}_{n=1}^{\infty}$ are equivalent there. Later - even without imposing any regularity or structure conditions - it was proved in [11] (with an important intermediate step [12]) that the general Franklin system is unconditional in $L^{p}[0,1], 1<p<\infty$, and equivalent to a carefully selected subsequence of the Haar system.

In this paper we apply the martingale approach of T. Figiel [6] to the general Franklin system and extend the results of [10] from the scalar case to that of UMD spaces. Recall that a Banach space $X$ has the UMD property if in $L^{p}(X), 1<p<\infty$, the vector-valued version of Burkholder's theorem holds true: If $\left\{d_{n}\right\}$ is a martingale difference sequence in $L^{p}(X), 1<p<\infty$, then

$$
\left\|\sum_{n=1}^{m} \pm d_{n}\right\|_{L^{p}(X)} \leq C_{p}\left\|\sum_{n=1}^{m} d_{n}\right\|_{L^{p}(X)}, \quad m \in \mathbb{N},
$$

where $C_{p}=C_{p}(X)$. Our reference for UMD spaces is [3].

Now, we formulate the main result of the paper:

TheOREM 2.1. Let $\left\{\mathcal{P}_{j}, j \geq 0\right\}$ be a sequence of quasi-dyadic partitions satisfying the weak regularity condition with parameter $\gamma>1$. Let $\left\{\phi_{n}, n \geq 0\right\}$ be the corresponding general Franklin system, normalized in $L^{\infty}$. Let $X$ be an $U M D$ space, and let $a_{n} \in X$. Then for each $1<p<\infty$,

$$
\frac{1}{C}\left\|\sum_{n=1}^{\infty} a_{n} h_{n}\right\|_{L^{p}(X)} \leq\left\|\sum_{n=1}^{\infty} a_{n} \phi_{n}\right\|_{L^{p}(X)} \leq C\left\|\sum_{n=1}^{\infty} a_{n} h_{n}\right\|_{L^{p}(X)},
$$

where the constant $C=C_{\gamma, p, X}$ depends on $\gamma, p$ and $X$.

Comment. Since the general Haar functions $\left\{h_{n}, n \geq 1\right\}$ form a martingale difference sequence, the UMD property of $X$ implies that $\sum_{n=1}^{\infty} a_{n} h_{n}$ converges unconditionally, if it converges at all. By (2.7), the same assertion holds for the general Franklin series $\sum_{n=0}^{\infty} a_{n} \phi_{n}$. 


\section{PROOF OF THEOREM 2.1}

The first step of T. Figiel's approach to biorthogonal systems consists in decomposing each individual Franklin function into a Haar series, rescaled to a suitable dyadic level. Thus T. Figiel reduces the norm estimates for series of Franklin functions to a sequence of norm estimates for basic operators, splitting and rearranging the Haar system. The special symmetry in the equations defining Figiel's basic operators reflects directly the obvious fact that each dyadic interval splits into two of equal length.

For quasi-dyadic partitions this symmetry does not hold any longer so that in this paper we are led to analyze certain weighted forms of Figiel's basic operators. These are the operators $T_{m}$ and $U_{m}$ defined below.

Let $\tau_{m}, m \in \mathbb{Z}$, be the rearrangement of $\mathcal{I}$ defined by

$$
\tau_{m}\left(I_{j, k}\right)=I_{j, k+m} \quad \text { when } \quad 1 \leq k, k+m \leq 2^{j} .
$$

Let $T_{m}$ denote the linear extension of a map given by

$$
T_{m} h_{I}=x_{I, \tau_{m}(I)} h_{\tau_{m}(I)} \quad \text { where } \quad\left|x_{I, \tau_{m}(I)}\right| \leq B \frac{|I|}{|I|+\left|\tau_{m}(I)\right|},
$$

and $B$ is a constant.

Theorem 3.1. Let $\left\{\mathcal{P}_{j}, j \geq 0\right\}$ be a sequence of quasi-dyadic partitions satisfying the weak regularity condition with parameter $\gamma$. Then there is $C>0$, depending on $X, p$ and $\gamma$, such that

$$
\left\|T_{m}: L^{p}(X) \rightarrow L^{p}(X)\right\| \leq C B \log (|m|+2),
$$

where $B$ is the constant from (3.2).

Define $U_{m}$ to be the linear extension of

$$
U_{m} h_{I}=y_{I, \tau_{m}(I)} g_{I, \tau_{m}(I)} \quad \text { where } \quad g_{I, \tau_{m}(I)}=1_{\tau_{m}(I)}-\frac{\left|\tau_{m}(I)\right|}{|I|} 1_{I},
$$

and

$$
\left|y_{I, \tau_{m}(I)}\right| \leq B \frac{|I|}{|I|+\left|\tau_{m}(I)\right|}
$$

TheOREM 3.2. Let $\left\{\mathcal{P}_{j}, j \geq 0\right\}$ be a sequence of quasi-dyadic partitions satisfying the weak regularity condition with parameter $\gamma$. Then there is $C>0$, depending on $X, p$ and $\gamma$, such that

$$
\left\|U_{m}: L^{p}(X) \rightarrow L^{p}(X)\right\| \leq C B \log (|m|+2),
$$

where $B$ is the constant from (3.3)-(3.4).

Throughout the paper we exploit the UMD property of $X$ using J. Bourgain's version of E. M. Stein's martingale inequality and J. P. Kahane's 
contraction principle. Recall that J. Bourgain's version of E. M. Stein's martingale inequality asserts that

$$
\int_{0}^{1}\left\|\sum r_{j}(t) \mathbb{E}_{j}\left(f_{j}\right)\right\|_{L^{p}(X)}^{p} d t \leq C_{p}(X) \int_{0}^{1}\left\|\sum r_{j}(t) f_{j}\right\|_{L^{p}(X)}^{p} d t,
$$

for any $f_{j} \in L^{p}(X)$ and any sequence $\left\{\mathbb{E}_{j}\right\}$ of conditional expectation operators corresponding to an increasing sequence of $\sigma$-algebras; here $\left\{r_{j}\right\}$ denotes the Rademacher functions. For $C_{p}(X)$ to be finite, that is to say, for (3.5) to hold, the Banach space $X$ has to satisfy the UMD property. The origin of (3.5) is J. Bourgain's paper [2], where it is employed to show boundedness of vector-valued, singular convolution operators. Later it played a decisive role in the analysis of vector-valued paraproduct operators that appeared in T. Figiel's proof of the T(1) theorem [7]. In [14] the inequality (3.5) was used to extend B. Maurey's isomorphism to the UMD case. J. Bourgain's proof of (3.5) is recorded in [8].

J.-P. Kahane's well known contraction principle asserts that in any Banach space $X$,

$$
\int_{0}^{1}\left\|\sum r_{j}(t) c_{j} x_{j}\right\|_{X}^{p} d t \leq C_{p} \sup _{j}\left|c_{j}\right|^{p} \int_{0}^{1}\left\|\sum r_{j}(t) x_{j}\right\|_{X}^{p} d t
$$

for any bounded numerical sequence $\left\{c_{j}\right\}$ and any $x_{j} \in X$; here again $\left\{r_{j}\right\}$ denotes the Rademacher system.

Now, we proceed as follows. First, we show how Theorem 2.1 follows from Theorems 3.1 and 3.2. This is done in Section 3.1. Then we give the proof of Theorems 3.1 and 3.2 in Section 3.2.

3.1. Figiel's non-standard expansion of $\phi_{n}$. In this section we apply Figiel's expansion to our (general) Franklin systems and show that it yields the proof of Theorem 2.1 assuming that Theorems 3.1 and 3.2 hold.

In order to prove (2.7), by a standard duality argument it suffices to verify the right-hand inequality of (2.7).

Next we write down Figiel's non-standard expansion of $\phi_{n}$ using general Haar functions. In the present context, Figiel's expansion is equation (3.11) below. Since [10] contains explicit information on the pointwise decay of $\phi_{n}$ 's (and consequently, also its derivatives), (3.11) forms the first step in the proof of Theorem 2.1.

Figiel's non-standard decomposition of $\phi_{j, k}$. Set $h_{j, k}^{*}=h_{j, k} /\left\|h_{j, k}\right\|_{1}$. It follows by the weak regularity of the sequence of partitions that $\left|h_{j, k}^{*}\right| \sim_{\gamma}$ $1 /\left|I_{j, k}\right|$ on $I_{j, k}$. Now, write 


$$
\phi_{j, k}=\sum_{l=1}^{2^{j}} \phi_{j, k} \cdot 1_{j, l}
$$

and further

$$
\phi_{j, k} \cdot 1_{j, l}=\alpha_{(j, k),(j, l)} 1_{j, l}+\sum_{\xi=0}^{\infty} \psi_{(j, k) ; l ; \xi}
$$

with

$$
\psi_{(j, k) ; l ; \xi}=\sum_{\eta: I_{j+\xi, \eta} \subset I_{j, l}} \beta_{(j, k),(\xi, \eta)} h_{j+\xi, \eta}
$$

and

$$
\alpha_{(j, k),(j, l)}=\frac{1}{\left|I_{j, l}\right|} \int_{I_{j, l}} \phi_{j, k}(s) d s, \quad \beta_{(j, k),(\xi, \eta)}=\int_{0}^{1} \phi_{j, k}(s) h_{j+\xi, \eta}^{*}(s) d s .
$$

Taking $\xi \geq 0$ and $m=l-k$, define

$$
\Psi_{\xi, m}\left(h_{j, k}\right)=\psi_{(j, k) ; m+k ; \xi} \quad \text { and } \quad V_{m} h_{j, k}=\alpha_{(j, k),(j, m+k)} 1_{j, m+k} .
$$

Clearly,

$$
\phi_{j, k}=\sum_{m} V_{m} h_{j, k}+\sum_{m} \sum_{\xi=0}^{\infty} \Psi_{\xi, m} h_{j, k} .
$$

By (3.11) we can reduce the right-hand inequality in (2.7) to norm estimates for the sequence of operators $V_{m}$ and $\Psi_{\xi, m}$. Precisely, for (2.7) to hold it is enough that

$$
\left\|\sum_{m} V_{m}\right\|_{L^{p}(X)}+\sum_{m} \sum_{\xi \geq 0}\left\|\Psi_{\xi, m}\right\|_{L^{p}(X)}<\infty .
$$

We obtain good estimates for the operators $\Psi_{\xi, m}$ and $V_{m}$ by using rather precise inequalities for the coefficients $\alpha_{(j, k),(j, l)}, \beta_{(j, k),(\xi, \eta)}$ appearing in Figiel's expansion. The inequalities are obtained from the next proposition (cf. Proposition 2.9 of [10]; the estimate for $\phi_{n}^{\prime}$ follows from a pointwise estimate for $\phi_{n}$, piecewise linearity of $\phi_{n}$ and weak regularity of the sequence of partitions).

Proposition 3.3. Let $\left\{\mathcal{P}_{j}, j \geq 0\right\}$ be a quasi-dyadic sequence of partitions satisfying the weak regularity condition with parameter $\gamma$. Let $\left\{\phi_{n}\right.$, $n \geq 0\}$ be the corresponding $L^{\infty}$-normalized Franklin system. Then:

(i) For each $j \geq 0,1 \leq k \leq 2^{j}$ and $n=2^{j}+k, \phi_{n}=\phi_{j, k}$ is linear on each interval $I_{j+1, l}, 1 \leq l \leq 2^{j+1}$.

(ii) For each $1 \leq p \leq \infty$ we have $\left\|\phi_{j, k}\right\|_{p} \sim_{\gamma}\left|I_{j, k}\right|^{1 / p}$. Moreover, $\phi_{n}\left(t_{n}\right) \sim_{\gamma} 1$, and $\int_{0}^{1} \phi_{n}(s) d s=0$ for $n \geq 1$. 
(iii) Pointwise estimates for $\phi_{j, k}$ and $\phi_{j, k}^{\prime}$ : there are $C_{\gamma}$ and $0<\theta<1$ such that for all $j$ and $1 \leq k, l \leq 2^{j}$,

$$
\begin{array}{ll}
\left|\phi_{j, k}(t)\right| \leq C_{\gamma} \theta^{|k-l|} \frac{\left|I_{j, k}\right|}{\left|I_{j, k}\right|+\operatorname{dist}\left(I_{j, k}, I_{j, l}\right)+\left|I_{j, l}\right|} & \text { for } t \in I_{j, l} . \\
\left|\phi_{j, k}^{\prime}(t)\right| \leq C_{\gamma} \frac{\theta^{|k-l|}}{\left|I_{j, l}\right|} \frac{\left|I_{j, k}\right|}{\left|I_{j, k}\right|+\operatorname{dist}\left(I_{j, k}, I_{j, l}\right)+\left|I_{j, l}\right|} & \text { for } t \in I_{j, l} .
\end{array}
$$

Next we obtain estimates for the coefficients $\alpha_{(j, k),(j, l)}$ and $\beta_{(j, k),(\xi, \eta)}$. We start with $\beta_{(j, k),(\xi, \eta)}$ using the Lipschitz properties of $\phi_{j, k}$. It follows from Proposition 3.3(iii), the condition $I_{j+\xi, \eta} \subset I_{j, l}$ and $\int_{0}^{1} h_{j+\xi, \eta}^{*}(u) d u=0$ that

$$
\begin{aligned}
\left|\beta_{(j, k),(\xi, \eta)}\right| & =\left|\int_{I_{j+\xi, \eta}}\left(\phi_{j, k}(u)-\phi_{j, k}\left(t_{j+\xi, \eta}\right)\right) h_{j+\xi, \eta}^{*}(u) d u\right| \\
& \leq C_{\gamma}\left|I_{j+\xi, \eta}\right| \sup _{u \in I_{j+\xi, \eta}}\left|\phi_{j, k}^{\prime}(u)\right| \\
& \leq C_{\gamma} \theta^{|k-l|} \frac{\left|I_{j+\xi, \eta}\right|}{\left|I_{j, l}\right|} \frac{\left|I_{j, k}\right|}{\left|I_{j, k}\right|+\operatorname{dist}\left(I_{j, k}, I_{j, l}\right)+\left|I_{j, l}\right|} .
\end{aligned}
$$

By weak regularity of the sequence of partitions (cf. (2.6)) we know that $\left|I_{j+\xi, \eta}\right| /\left|I_{j, l}\right| \leq(\gamma /(\gamma+1))^{\xi}$. Thus we get

$$
\left|\beta_{(j, k),(\xi, \eta)}\right| \leq C_{\gamma} \theta^{|k-l|}\left(\frac{\gamma}{\gamma+1}\right)^{\xi} \frac{\left|I_{j, k}\right|}{\left|I_{j, k}\right|+\left|I_{j, l}\right|},
$$

and consequently

$$
\left|\psi_{(j, k) ; l ; \xi}\right| \leq C_{\gamma} \theta^{|k-l|}\left(\frac{\gamma}{\gamma+1}\right)^{\xi} \frac{\left|I_{j, k}\right|}{\left|I_{j, k}\right|+\left|I_{j, l \mid}\right|}\left|h_{j, l}\right| .
$$

Similarly, by using Proposition 3.3(ii), (iii) we find that

$$
\left|\alpha_{(j, k),(j, l)}\right| \leq C_{\gamma} \theta^{|k-l|} \frac{\left|I_{j, k}\right|}{\left|I_{j, k}\right|+\left|I_{j, l}\right|} \quad \text { and } \quad \sum_{l=1}^{2^{j}}\left|I_{j, l}\right| \alpha_{(j, k),(j, l)}=0 .
$$

Having obtained the inequalities for $\alpha_{(j, k),(j, l)}, \beta_{(j, k),(\xi, \eta)}$, we now turn to the norm estimates for $V_{m}$ and $\Psi_{\xi, m}$. These are based on the estimates for $T_{m}$ and $U_{m}$ established in Theorems 3.1 and 3.2.

The operators $\Psi_{\xi, m}$. Consider the operator $T_{m}$ given by

$$
T_{m} h_{j, k}=\frac{\left|I_{j, k}\right|}{\left|I_{j, k}\right|+\left|I_{j, m+k}\right|} h_{j, m+k} .
$$

It follows by (3.13) that

$$
\left|\Psi_{\xi, m} h_{j, k}\right| \leq C_{\gamma} \theta^{|m|}\left(\frac{\gamma}{\gamma+1}\right)^{\xi}\left|T_{m} h_{j, k}\right| .
$$


For fixed $\xi$ and $m$, the sequence $\left\{\Psi_{\xi, m} h_{j, k}\right\}_{(j, k)}$ in lexicographical order is a martingale difference sequence. Let $a_{j, k} \in X$. Hence by the UMD property of $X$,

$$
\left\|\sum_{j, k} a_{j, k} \Psi_{\xi, m} h_{j, k}\right\|_{L^{p}(X)} \sim_{X, p}\left\|\sum_{j, k} \pm a_{j, k} \Psi_{\xi, m} h_{j, k}\right\|_{L^{p}(X)} .
$$

Averaging over \pm , using (3.15) and applying Kahane's contraction principle we find that

$$
\left\|\sum_{j, k} a_{j, k} \Psi_{\xi, m} h_{j, k}\right\|_{L^{p}(X)} \leq C_{\gamma, p, X} \theta^{|m|}\left(\frac{\gamma}{\gamma+1}\right)^{\xi}\left\|\sum_{j, k} a_{j, k} T_{m} h_{j, k}\right\|_{L^{p}(X)} .
$$

Combining this with Theorem 3.1 we get

$$
\sum_{\xi, m}\left\|\Psi_{\xi, m}\right\|_{L^{p}(X)} \leq C_{\gamma, p, X} \sum_{\xi, m} \theta^{|m|}\left(\frac{\gamma}{\gamma+1}\right)^{\xi} \log (2+|m|)<\infty .
$$

The operators $V_{m}$. Consider the operators $U_{m}$ given by

$$
U_{m} h_{j, k}=\alpha_{(j, k),(j, m+k)}\left(1_{j, m+k}-\frac{\left|I_{j, m+k}\right|}{\left|I_{j, k}\right|} 1_{j, k}\right) .
$$

It follows by (3.14) that

$$
\sum_{m} V_{m} h_{j, k}=\sum_{m} U_{m} h_{j, k}
$$

Combining this with estimates from (3.14) and Theorem 3.2 we get

$$
\begin{aligned}
\left\|\sum_{m} \sum_{j, k} a_{j, k} V_{m} h_{j, k}\right\|_{L^{p}(X)} & \leq \sum_{m}\left\|\sum_{j, k} a_{j, k} U_{m} h_{j, k}\right\|_{L^{p}(X)} \\
& \leq C_{\gamma, p, X} \sum_{m} \theta^{|m|} \log (2+|m|)\left\|\sum_{j, k} a_{j, k} h_{j, k}\right\|_{L^{p}(X)} \\
& \leq C_{\gamma, p, X}\left\|\sum_{j, k} a_{j, k} h_{j, k}\right\|_{L^{p}(X)} .
\end{aligned}
$$

Combining this with (3.16) we get (3.12), and consequently the right-hand inequality in (2.7).

3.2. Boundedness of the rearrangement operators. In this section we prove the norm estimates for the operators $T_{m}$. By combinatorial means we provide a reduction to estimates for $X$-valued martingale differences, and we control the latter using J. Bourgain's version of E. M. Stein's martingale inequality and the contraction principle of J.-P. Kahane. The basic strategy for proving $\left\|T_{m}\right\|_{p} \leq C_{\gamma, p, X} \log (|m|+2)$ consists in splitting $\mathcal{I}$ into at most $C \log (|m|+2)$ collections $\mathcal{F}$ such that $T_{m}$ restricted to each of them is bounded independently of $m$. 
3.2.1. A rough decomposition of $\mathcal{I}$. As in [6], we start by separating the orbit of $\tau_{m}$ and the levels of $\mathcal{I}$.

For convenience, we introduce the following terminology: let $K=$ $\bigcup_{k \in M} I_{j, k}, L=\bigcup_{l \in P} I_{j, l}$, where $M, P \subset\left\{1, \ldots, 2^{j}\right\}$, and the sets $M, P$ are such that

$$
|k-l|>1 \quad \text { for all } k \in M, l \in P,
$$

then we say that there is a gap of one $\mathcal{I}_{j}$-interval between $K$ and $L$.

Let $\xi_{m}$ be the first level where $\tau_{m}$ is defined.

Proposition 3.4. There exists a decomposition of $\mathcal{I}$ into pairwise disjoint collections

$$
\left\{\mathcal{F}^{(s, r)}: s \leq \kappa-1, r \leq r_{0}\right\}
$$

where $\kappa \sim \log (|m|+2), r_{0} \leq 8$, so that:

(i) If $I \in \mathcal{F}^{(s, r)}$, then $\tau_{m}(I) \notin \mathcal{F}^{(s, r)}$.

(ii) If $I, J \in \mathcal{F}^{(s, r)}, I \in \mathcal{I}_{i}, J \in \mathcal{I}_{j}$ and $I \subsetneq J$, then $i \geq j+\kappa$.

(iii) If $I, J \in \mathcal{F}^{(s, r)} \cap \mathcal{I}_{j}$ then

$$
\operatorname{dist}\left(I \cup \tau_{m}(I), J \cup \tau_{m}(J)\right)>0 .
$$

REMARK 3.5. By modifying the decomposition outlined below we can achieve that in addition to (3.17) we also have

$$
\operatorname{dist}\left(I \cup \tau_{m \pm 1}(I), J \cup \tau_{m \pm 1}(J)\right)>0 .
$$

Proof. We start by splitting each of the $\mathcal{I}_{j}$ with $j \geq \xi_{m}$ into disjoint families $\mathcal{I}_{j, r}, 1 \leq r \leq 8$, with the following properties:

(a) If $I_{j, k} \in \mathcal{I}_{j, r}$, then $\tau\left(I_{j, k}\right)=I_{j, k+m} \notin \mathcal{I}_{j, r}$.

(b) If $I_{j, k}, I_{j, l} \in \mathcal{I}_{j, r}, k \neq l$, then $|k-l|>1,|k+m-l|>1$ and $|l+m-k|>1$. In other words: there is a gap of one $\mathcal{I}_{j}$-interval between $I_{j, k} \cup \tau\left(I_{j, k}\right)$ and $I_{j, l} \cup \tau\left(I_{j, l}\right)$. Notice that this condition means that $I_{j, k}$ is not a neighbour of $I_{j, l}$ or $\tau\left(I_{j, l}\right)$. Notice also that only in case $|m|=1$ does it happen that $I_{j, k}$ is a neighbour of $\tau\left(I_{j, k}\right)$.

We get the splitting of $\mathcal{I}_{j}$ into the families $\mathcal{I}_{j, r}$ as follows:

(1) Split each $\mathcal{I}_{j}$ into the odd numbered intervals

$$
O_{j}=\left\{I_{j, 2 k^{\prime}+1}: 1 \leq 2 k^{\prime}+1 \leq 2^{j}\right\}
$$

and into the even numbered intervals

$$
E_{j}=\left\{I_{j, 2 k^{\prime}}: 1 \leq 2 k^{\prime} \leq 2^{j}\right\} .
$$

Both these families have the property that if an interval is in one of these families, then its neighbours are in the other family. Now we further decompose the families $O_{j}, E_{j}$. Here the construction distinguishes between the case when $m$ is odd or even. 
(2) Let $m$ be even. Consider first $O_{j}$. We decompose $O_{j}$ along the orbit of $\tau_{m}$. Put

$$
\begin{aligned}
& O_{m ; j, 1}=\left\{\tau_{m \cdot(2 n)}(I): n \in \mathbb{Z}, I=I_{j, 2 k^{\prime}+1} \text { with } 1 \leq 2 k^{\prime}+1 \leq|m|\right\} \\
& O_{m ; j, 2}=\left\{\tau_{m \cdot(2 n+1)}(I): n \in \mathbb{Z}, I=I_{j, 2 k^{\prime}+1} \text { with } 1 \leq 2 k^{\prime}+1 \leq|m|\right\},
\end{aligned}
$$

Clearly each of the collections $O_{m ; j, 1}, O_{m ; j, 2}$ satisfies (i) and (iii).

By the same procedure we decompose $E_{j}$ along the orbits of $\tau_{m}$ obtaining collections $E_{m ; j, 1}, E_{m ; j, 2}$ that satisfy (i) and (iii). Thus $E_{m ; j, 1}, E_{m ; j, 2}, O_{m ; j, 1}$, $O_{m ; j, 2}$ is the required splitting of $\mathcal{I}_{j}$ for $m$ even.

(3) For $m$ odd with $|m|>1, O_{j}$ and $E_{j}$ satisfy (i), but not (iii). Clearly $m-1$ and $m+1$ are even. Define the splitting of $O_{j}$ into $O_{m+1 ; j, 1}, O_{m+1 ; j, 2}$ and simultaneously split $O_{j}$ into $O_{m-1 ; j, 1}, O_{m-1 ; j, 2}$. Then form the intersections

$$
R_{m ; j, \alpha, \beta}=O_{m+1 ; j, \alpha} \cap O_{m-1 ; j, \beta}, \quad \alpha, \beta \in\{1,2\} .
$$

Each of the collections $R_{m ; j, \alpha, \beta}, \alpha, \beta \in\{1,2\}$, satisfies (i) and (iii). Thus $\left\{R_{m ; j, \alpha, \beta}, \alpha, \beta \in\{1,2\}\right\}$ forms the required splitting of $O_{j}$. The decomposition of $E_{j}$ is defined analogously.

(4) For $|m|=1$, take the splitting of $\mathcal{I}_{j}$ according to $k \bmod 3$.

Then take $\kappa=\kappa_{m}, \kappa \sim \log (|m|+2)$. Write each $j \geq \xi_{m}$ as $j=\xi_{m}+\kappa l+s$, $l \geq 0,0 \leq s \leq \kappa-1$. For fixed $r, s$, let

$$
\begin{aligned}
& \mathcal{B}_{l}=\mathcal{B}_{l}^{(r, s)}=\mathcal{I}_{\xi_{m}+\kappa l+s, r} \\
& \mathcal{F}=\mathcal{F}^{(s, r)}=\bigcup_{l \geq 0} \mathcal{B}_{l} .
\end{aligned}
$$

3.2.2. Constructing nested collections. Let $\mathcal{F}$ be one of the families obtained in Proposition 3.4. We show below that $T_{m}$ restricted to $\mathcal{F}$ is bounded. We will do this by relating $\left\{I \cup \tau_{m}(I): I \in \mathcal{F}\right\}$ to a nested collection of measurable sets $\{A(I): I \in \mathcal{F}\}$, so that $T_{m}$ can be controlled by a martingale transform acting on $\left\{1_{A(I)}: I \in \mathcal{F}\right\}$. Thus the construction of a nested family $\{A(I): I \in \mathcal{F}\}$ so that $A(I)$ stays close to $I \cup \tau_{m}(I)$ is crucial for our proof that $T_{m}$ is bounded.

TheOREM 3.6. Let $m \in \mathbb{Z}$. Let $\tau=\tau_{m}$. Let $\mathcal{F} \subseteq \mathcal{I}$ be one of the families obtained in Proposition 3.4. Then for every $I \in \mathcal{F}$ there exists $A(I) \subseteq[0,1]$ so that

$$
I \cup \tau(I) \subseteq A(I) \subseteq\left(I^{-} \cup I \cup I^{+}\right) \cup\left(\tau(I)^{-} \cup \tau(I) \cup \tau(I)^{+}\right),
$$

and $\{A(I): I \in \mathcal{F}\}$ is a nested collection of sets in the sense that

$$
A(I) \cap A(J) \neq 0 \quad \text { implies } \quad A(I) \subseteq A(J) \text { or } A(J) \subseteq A(I) .
$$


Proof. We first define $A(I)$ through an inductive procedure. Later we will verify (3.21) and (3.22). For convenience, let

$$
j_{l}=\xi_{m}+\kappa l+s, \quad \text { where } \mathcal{F}=\mathcal{F}^{(s, r)} .
$$

The $A(K, l)$ operation. Let $K=\bigcup_{\mu \in M} I_{j_{l-1}, \mu}$, where $M \subset\left\{1, \ldots, 2^{j_{l-1}}\right\}$. For such $K$, define

$$
\begin{aligned}
& C(K, l)=\left\{I \in \mathcal{B}_{l}: \operatorname{dist}(I \cup \tau(I), K)=0\right\}, \\
& A(K, l)=K \cup \bigcup_{I \in C(K, l)}(I \cup \tau(I)) .
\end{aligned}
$$

Note that $I \in C(K, l)$ iff either $I \subset K$, or $\tau(I) \subset K$, or one of $I, \tau(I)$ has a common endpoint with one of $I_{j_{l-1}, \mu}, \mu \in M$. Clearly $A(K, l)$ can be written as a union of intervals in $\mathcal{I}_{j_{l}}$.

Defining the atom $A(I)$. Let $I \in \mathcal{F}$. Take $l \geq 0$ such that $I \in \mathcal{B}_{l}$ (there is only one such $l$ ). Then define inductively:

$$
\begin{aligned}
A_{l}(I) & =I \cup \tau(I), \\
A_{n+1}(I) & =A\left(A_{n}(I), n+1\right) \quad \text { for } \quad n \geq l, \\
A(I) & =\bigcup_{n \geq l} A_{n}(I) .
\end{aligned}
$$

Observe that each $A_{n}(I)$ is a union of some intervals from $\mathcal{I}_{j_{n}}$. Hence the operation $A(\cdot, n+1)$ can be applied to $A_{n}(I)$ so that (at least) $A(I)$ is a well defined measurable subset of the unit interval. Moreover it is immediate that

$$
A_{n}(I) \subset A_{n+1}(I) .
$$

We start with a simple fact displaying the basic properties of the $A(K, l)$ operation.

FACT 3.7. Let $J \in \mathcal{B}_{l}$. Then either $J \in C(K, l)$, or there is a gap of at least one $\mathcal{I}_{j_{l}}$-interval between $J$ and $A(K, l)$, and between $\tau(J)$ and $A(K, l)$.

Proof. Suppose that $J \notin C(K, l)$. Let $\Delta \in \mathcal{I}_{j_{l}}, \Delta \subset A(K, l)$. It is enough to check that there is a gap of one $\mathcal{I}_{j_{l}}$-interval between $J$ and $\Delta$, and between $\tau(J)$ and $\Delta$. That is, $J, \tau(J) \neq \Delta, \Delta^{-}, \Delta^{+}$.

Consider the following cases:

(1) $\Delta \subset K$. As $K$ is a union of intervals from the level $\mathcal{I}_{j_{l-1}}$, there is $\widetilde{\Delta} \in \mathcal{I}_{j_{l-1}}$ such that $\Delta \subset \widetilde{\Delta} \subset K$. Note that $J \neq \Delta$ and $\tau(J) \neq \Delta-$ otherwise, we would have $J \in C(K, l)$. Next, if $J=\Delta^{-}, \Delta^{+}$, then either $J \subset \widetilde{\Delta}$, or $J$ and $\widetilde{\Delta}$ have a common endpoint-but then $J \in C(K, l)$; consequently, $J \neq \Delta^{-}, \Delta^{+}$. An analogous argument gives $\tau(J) \neq \Delta^{-}, \Delta^{+}$. 
(2) $\Delta \not \subset K$, but $\Delta \in C(K, l)$. Then $\Delta \in \mathcal{B}_{l}$. Clearly, $J \neq \Delta$, and by condition (i) for the splittings $\mathcal{I}_{j, r}$ (see Proposition 3.4), $\tau(J) \neq \Delta$. By condition (iii) for the splittings $\mathcal{I}_{j, r}$, we get $J, \tau(J) \neq \Delta^{-}, \Delta^{+}$.

(3) $\Delta \not \subset K$ and $\Delta \notin C(K, l)$. Then there is $I \in C(K, l)$ such that $\Delta=\tau(I)$. Observe that $I \in \mathcal{B}_{l}$, and therefore there must be $J \neq I$ and $\tau(J) \neq \tau(I)=\Delta$. Further, $J \neq \Delta$ by condition (i) for splittings, and $J, \tau(J) \neq \Delta^{-}, \Delta^{+}$by (iii).

The next lemma is crucial. In $\mathcal{T}_{n}$ (defined below) we gather the $n$th approximation to the first $n$ generations of the sets $\{A(I): I \in \mathcal{F}\}$. The conclusion of Lemma 3.8 asserts that not only is $\mathcal{T}_{n}$ a nested collection of sets but also disjoint sets in $\mathcal{T}_{n}$ have a strong separation property.

LEMma 3.8. For $n \geq 0$, let

$$
\mathcal{T}_{n}=\left\{A_{n}(I): I \in \mathcal{B}_{l}, 0 \leq l \leq n\right\} .
$$

Let $K, L \in \mathcal{T}_{n}$. Then either $K \subset L$, or $L \subset K$, or there is a gap of at least one $\mathcal{I}_{j_{n}}$-interval between $K$ and $L$.

Proof. The proof is by induction with respect to $n$.

STEP $n=0$. For $n=0$ we have

$$
\mathcal{T}_{0}=\left\{A_{0}(I): I \in \mathcal{B}_{0}\right\} \quad \text { and } \quad A_{0}(I)=I \cup \tau(I) \quad \text { for } I \in \mathcal{B}_{0} .
$$

It follows from Proposition 3.4(iii) that if $I, J \in \mathcal{B}_{0}, I \neq J$, then $A_{0}(I), A_{0}(J)$ are disjoint, and there is a gap of at least one $\mathcal{I}_{j_{0}}$-interval between $A_{0}(I)$ and $A_{0}(J)$.

STEP $n+1$. Suppose now that the conclusion of Lemma 3.8 is satisfied for $n$; we check it for $n+1$. It follows from the definition of $A_{n}(\cdot)$ and $\mathcal{T}_{n+1}$ that

$$
\begin{aligned}
\mathcal{T}_{n+1} & =\left\{A_{n+1}(I): I \in \mathcal{B}_{l}, 0 \leq l \leq n\right\} \cup\left\{A_{n+1}(I): I \in \mathcal{B}_{n+1}\right\} \\
& =\left\{A\left(K^{\prime}, n+1\right): K^{\prime} \in \mathcal{T}_{n}\right\} \cup\left\{I \cup \tau(I): I \in \mathcal{B}_{n+1}\right\} .
\end{aligned}
$$

Let $K, L \in \mathcal{T}_{n+1}$. Consider the following cases:

(1) $K=I \cup \tau(I), L=J \cup \tau(J)$ with $I, J \in \mathcal{B}_{n+1}$.

If $I=J$, then $K=L$. If $I \neq J$, then $K \not \subset L, L \not \subset K$, and the required separation of $K$ and $L$ follows from Proposition 3.4(iii).

(2) $K=A\left(K^{\prime}, n+1\right)$ with $K^{\prime} \in \mathcal{T}_{n}$ and $L=J \cup \tau(J)$ with $J \in \mathcal{B}_{n+1}$.

In this case, if $J \in C\left(K^{\prime}, n+1\right)$, then by the definition of $A(\cdot, n+1)$ we have $L=J \cup \tau(J) \subset A\left(K^{\prime}, n+1\right)=K$. If $J \notin C\left(K^{\prime}, n+1\right)$, then the separation of $K$ and $L$ by at least one $\mathcal{I}_{j_{n+1}}$-interval is a consequence of Fact 3.7.

(3) $L=A\left(L^{\prime}, n+1\right)$ with $L^{\prime} \in \mathcal{T}_{n}$ and $K=I \cup \tau(I)$ with $I \in \mathcal{B}_{n+1}$.

This case is symmetric to $(2)$. 
(4) $K=A\left(K^{\prime}, n+1\right)$ and $L=A\left(L^{\prime}, n+1\right)$ with $K^{\prime}, L^{\prime} \in \mathcal{T}_{n}$.

By the induction hypothesis, either $K^{\prime} \subset L^{\prime}$, or $L^{\prime} \subset K^{\prime}$, or there is a gap of at least one $\mathcal{I}_{j_{n}}$-interval between $K^{\prime}$ and $L^{\prime}$. Consider now the following subcases:

(4a) $K^{\prime} \subset L^{\prime}$.

The inclusion $K^{\prime} \subset L^{\prime}$ and the definition of $C(\cdot, n+1)$ imply that $C\left(K^{\prime}\right.$, $n+1) \subset C\left(L^{\prime}, n+1\right)$. Consequently, $K=A\left(K^{\prime}, n+1\right) \subset A\left(L^{\prime}, n+1\right)=L$.

(4b) $L^{\prime} \subset K^{\prime}$.

By an argument as in (4a), $L \subset K$.

(4c) There is a gap of at least one $\mathcal{I}_{j_{n}}$-interval between $K^{\prime}$ and $L^{\prime}$.

Let $I, J \in \mathcal{I}_{j_{n+1}}, I \subset K, J \subset L$. We check that there is a gap of at least one $\mathcal{I}_{j_{n+1}}$-interval between $I$ and $J$.

Consider first $I$ and $K^{\prime}$. It follows by the definitions of $A(\cdot, n+1)$ and $C(\cdot, n+1)$ that there is $\widetilde{I} \in \mathcal{I}_{j_{n}}$ with $\widetilde{I} \subset K^{\prime}$ such that one of the following holds:

- $I \subset \widetilde{I}$, or $I \in \mathcal{B}_{n+1}$ and $\tau(I) \subset \widetilde{I}$,

- $I \in \mathcal{B}_{n+1}$, and either $I$ or $\tau(I)$ has a common endpoint with $\widetilde{I}$,

- $I=\tau\left(I^{\prime}\right)$ where $I^{\prime} \in \mathcal{B}_{n+1}$ and either $I^{\prime} \subset \widetilde{I}$, or one of $I^{\prime}, I=\tau\left(I^{\prime}\right)$ and $\widetilde{I}$ have a common endpoint.

Recall that $j_{n+1}=j_{n}+\kappa$. Therefore, setting $\widetilde{I}=\left[t_{j_{n}, k-1}, t_{j_{n}, k}\right]=\left[t_{j_{n+1}, 2^{\kappa}(k-1)}\right.$, $\left.t_{j_{n+1}, 2^{\kappa} k}\right]$, we find that in each of the above cases

$$
I \subset\left[t_{j_{n+1}, 2^{\kappa}(k-1)-|m|-1}, t_{j_{n+1}, 2^{\kappa} k+|m|+1}\right] .
$$

By similar considerations, setting $\widetilde{J}=\left[t_{j_{n}, l-1}, t_{j_{n}, l}\right]$, we find that

$$
J \subset\left[t_{j_{n+1}, 2^{\kappa}(l-1)-|m|-1}, t_{j_{n+1}, 2^{\kappa} l+|m|+1}\right] .
$$

The induction hypothesis implies that $|l-k|>1$; without loss of generality, suppose that $l \geq k+2$. Then by the choice of $\kappa$ we have

$$
\begin{aligned}
\left(2^{\kappa}(l-1)-|m|-1\right)-\left(2^{\kappa} k+|m|+1\right) & =2^{\kappa}(l-k-1)-2(|m|+1) \\
& \geq 2^{\kappa}-2(|m|+1)>1 .
\end{aligned}
$$

This implies that there is a gap of at least one $\mathcal{I}_{j_{n+1}}$-interval between $I$ and $J$.

Having analyzed the inductive procedures defining $A(I)$ we continue with the proof of Theorem 3.6. The following proposition contains the verification of (3.22).

Proposition 3.9. Let $I, J \in \mathcal{F}$. Then either $A(I) \subset A(J)$, or $A(J) \subset$ $A(I)$, or $A(I) \cap A(J)=\emptyset$. 
Comment. The proof below yields more precise statements. If $I, J \in \mathcal{B}_{l}$, $I \neq J$, then $A(I) \cap A(J)=\emptyset$. If $I \in \mathcal{B}_{l_{1}}$ and $J \in \mathcal{B}_{l_{2}}$ with $l_{1}<l_{2}$, then either $A(J) \subset A(I)$, or $A(I) \cap A(J)=\emptyset$.

Proof. In the proof below, we refer to cases considered in the proof of Lemma 3.8 above.

First, let $I, J \in \mathcal{B}_{l}, I \neq J$. By (1), there is a gap of one $\mathcal{I}_{j_{l}}$-interval between $A_{l}(I)$ and $A_{l}(J)$. Then, by an inductive argument and (4c), for each $n>l$, there is a gap of one $\mathcal{I}_{j_{n}}$-interval between $A_{n}(I)$ and $A_{n}(J)$. This in particular implies that these sets are disjoint for each $n \geq l$. As both $\left\{A_{n}(I), n \geq l\right\}$ and $\left\{A_{n}(J), n \geq l\right\}$ are increasing sequences of sets, this implies that $A(I) \cap A(J)=\emptyset$.

Consider now the case when $I \in \mathcal{B}_{l_{1}}$ and $J \in \mathcal{B}_{l_{2}}$ with $l_{1}<l_{2}$. Then there are two cases.

If $J \in C\left(A_{l_{2}-1}(I), l_{2}\right)$, then by (2) we have $A_{l_{2}}(J) \subset A_{l_{2}}(I)$, and by (4a) and an inductive argument, $A_{n}(J) \subset A_{n}(I)$ for all $n \geq l_{2}$. This and the definition of $A(\cdot)$ imply that $A(J) \subset A(I)$.

If $J \notin C\left(A_{l_{2}-1}(I), l_{2}\right)$, then by (2) there is a gap of one $\mathcal{I}_{j_{l_{2}}}$-interval between $A_{l_{2}}(I)$ and $A_{l_{2}}(J)$. Then by (4c) and an inductive argument, there is a gap of one $\mathcal{I}_{j_{n}}$-interval between $A_{n}(I)$ and $A_{n}(J)$, for each $n \geq l_{2}$. By an argument analogous to the above, this implies that $A(I) \cap A(J)=\emptyset$.

Having established that $\{A(I): I \in \mathcal{F}\}$ is a nested collection it remains to see that each $A(I)$ is a sufficiently good approximation of $I \cup \tau(I)$. This is the content of (3.21).

Proposition 3.10. For $I \in \mathcal{F}$, let

$$
\mathcal{S}(I)=I^{-} \cup I \cup I^{+} \cup \tau(I)^{-} \cup \tau(I) \cup \tau(I)^{+} .
$$

Then $I \cup \tau(I) \subset A(I) \subset \mathcal{S}(I)$.

Proof. For an interval $\Delta \in \mathcal{I}_{j_{l}}$, let

$$
A_{l}^{\prime}(\Delta)=\Delta, \quad A_{n+1}^{\prime}(\Delta)=A\left(A_{n}^{\prime}(\Delta), n+1\right) \quad \text { for } n \geq l .
$$

Observe that for $I \in \mathcal{B}_{l}$ and each $n \geq l$ we have $A_{n}(I)=A_{n}^{\prime}(I) \cup A_{n}^{\prime}(\tau(I))$ (proof by induction). Therefore, to prove the proposition, it is enough to check that for each $\Delta \in \mathcal{I}_{j_{l}}$ and $n \geq l$,

$$
A_{n}^{\prime}(\Delta) \subset \Delta^{-} \cup \Delta \cup \Delta^{+} \text {. }
$$

Let $k$ be such that

$$
\Delta=I_{j_{l}, k}=\left[t_{j_{l}, k-1}, t_{j_{l}, k}\right] .
$$

Then

$$
\Delta^{-} \cup \Delta \cup \Delta^{+}=\left[t_{j_{l}, k-2}, t_{j_{l}, k+1}\right]=\left[t_{j_{n}, 2^{\kappa(n-l)}(k-2)}, t_{j_{n}, 2^{\kappa(n-l)}(k+1)}\right] .
$$


Define

$$
\begin{array}{lll}
\eta_{l}=k-1, & \eta_{n+1}=2^{\kappa} \eta_{n}-|m|-1 & \text { for } n \geq l, \\
\xi_{l}=k, & \xi_{n+1}=2^{\kappa} \xi_{n}+|m|+1 & \text { for } n \geq l .
\end{array}
$$

By an inductive argument, essentially repeating the argument used already in $(4 \mathrm{c})$ of the proof of Lemma 3.8 we find that

$$
A_{n}^{\prime}(\Delta) \subset\left[t_{j_{n}, \eta_{n}}, t_{j_{n}, \xi_{n}}\right]
$$

Moreover, by an inductive argument we check that

$$
\eta_{n}-2^{\kappa(n-l)}(k-2) \geq 1 \quad \text { and } \quad 2^{\kappa(n-l)}(k+1)-\xi_{n} \geq 1 \quad \text { for } n \geq l .
$$

Let us check this for the sequence $\xi_{n}$ : for $n=l$ inequality (3.26) is satisfied. For $n \geq l$, using the definition of $\kappa$ we get

$$
\begin{aligned}
2^{\kappa(n+1-l)}(k+1)-\xi_{n+1} & =2^{\kappa(n+1-l)}(k+1)-\left(2^{\kappa} \xi_{n}+|m|+1\right) \\
& =2^{\kappa}\left(2^{\kappa(n-l)}(k+1)-\xi_{n}\right)-(|m|+1) \\
& \geq 2^{\kappa}-(|m|+1)>1 .
\end{aligned}
$$

Combining (3.25) and (3.26) we get

$$
A_{n}^{\prime}(\Delta) \subset \Delta^{-} \cup \Delta \cup \Delta^{+} \text {for all } n \geq l
$$

3.2.3. Proof of Theorem 3.1. Let $T=T_{m}$ be defined by (3.2). We consider only the case where $m>0$. The case $m<0$ is symmetric and can be treated analogously. We define two auxiliary mappings $\varrho_{1}$ and $\varrho_{2}$ acting on the intervals of $\mathcal{I}$, and hence on the associated general Haar functions.

Definition of $\varrho_{1}$ : Take $\Delta \in \mathcal{I}_{j}$, let $\Delta_{\text {left }}, \Delta_{\text {right }} \in \mathcal{I}_{j+1}$ be its "children", $\Delta_{\text {left,left }}, \Delta_{\text {left,right }} \in \mathcal{I}_{j+2}$ the children of $\Delta_{\text {left }}$, and $\Delta_{\text {right,left }}, \Delta_{\text {right,right }} \in$ $\mathcal{I}_{j+2}$ the children of $\Delta_{\text {right }}$. Now put

$$
\varrho_{1}\left(h_{\Delta}\right)=h_{\Delta_{\text {right,left }}} .
$$

Definition of $\varrho_{2}$ : Let $\Delta \in \mathcal{I}_{j}$, define

$$
\varrho_{2}\left(h_{\Delta_{\text {left,right }}}\right)=h_{\Delta},
$$

and set $\varrho_{2}$ equal to 0 for the Haar functions corresponding to the remaining three "grandchildren" of $\Delta$. Define

$$
Z\left(h_{I}\right)= \begin{cases}T_{4 m-1}\left(h_{I}\right) & \text { when } I=\Delta_{\text {right,left }} \text { for some } \Delta \\ 0 & \text { otherwise. }\end{cases}
$$

Observe that

$$
T_{m}=\varrho_{2} \circ Z \circ \varrho_{1} .
$$

Note that $\left\|\varrho_{1}\right\|_{L^{p}(X)},\left\|\varrho_{2}\right\|_{L^{p}(X)} \leq C_{p, \gamma}$ (by E. M. Stein's martingale inequality and weak regularity of the sequence of partitions). Therefore, it remains to check that

$$
\|Z\|_{L^{p}(X)} \leq C \log (|m|+2) .
$$


Next apply Proposition 3.4 with parameter $4 m-1$ and rearrangement $\tau_{4 m-1}$. Let $\left\{\mathcal{F}^{(r, s)}\right\}$ denote the resulting splitting. Clearly the total number of pairs $(r, s)$ is $\sim \kappa_{4 m-1} \sim \log (|m|+2)$. Fix $(r, s)$ and put $\mathcal{F}=\mathcal{F}^{(r, s)}$. The estimate (3.27) follows from our next claim: the linear extension of

$$
h_{I} \mapsto Z\left(h_{I}\right), \quad I \in \mathcal{F},
$$

has $L^{p}(X)$ norm bounded by $C_{p}$, where $C_{p}$ is independent of $m$. Recall that we obtained $\mathcal{F}$ by applying Proposition 3.4 with $4 m-1$ and $\tau_{4 m-1}$. Next apply Theorem 3.6 to $\mathcal{F}$ (with parameter $4 m-1$ and rearrangement $\left.\sigma=\tau_{4 m-1}\right)$. Let $\{A(I): I \in \mathcal{F}\}$ be the resulting nested collection. Next define $\mathcal{F}^{\prime}=\mathcal{F} \cap \mathcal{I}_{\text {right,left }}$. The following estimate is crucial:

$$
|A(I)| \sim_{\gamma}|I|+|\sigma(I)|, \quad I \in \mathcal{F}^{\prime}
$$

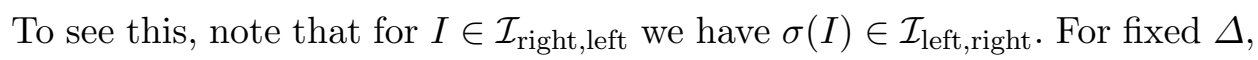
we have

$$
\begin{aligned}
& \Delta_{\text {right,left }}^{-} \cup \Delta_{\text {right,left }} \cup \Delta_{\text {right,left }}^{+} \subset \Delta, \\
& \Delta_{\text {left,right }}^{-} \cup \Delta_{\text {left,right }} \cup \Delta_{\text {left,right }}^{+} \subset \Delta .
\end{aligned}
$$

Now Proposition 3.10 and weak regularity imply (3.29).

By (3.29) we may rewrite the action of $Z$ restricted to $\mathcal{F}^{\prime}$. Let $\sigma=\tau_{4 m-1}$. Then

(3.30) $\quad Z\left(h_{I}\right)=z_{I, \sigma(I)} h_{\sigma(I)} \quad$ where $\quad\left|z_{I, \sigma(I)}\right| \leq C \frac{|I|}{|\sigma(I)|+|I|}, \quad I \in \mathcal{F}^{\prime}$.

It remains to fully exploit the content of (3.30) and turn it into a norm estimate for the operator $Z$. The estimates for $Z$ will be obtained as an application of E. M. Stein's martingale inequality. To prepare for its application we let $\mathcal{H}_{n}$ be the algebra generated by the atoms $A(I)$ with $I \in \mathcal{F}^{\prime} \cap \bigcup_{i \leq j_{n}} \mathcal{I}_{i}$, and let $E_{n}$ be the conditional expectation with respect to $\mathcal{H}_{n}$. For $I \in \mathcal{F}^{\prime} \cap \mathcal{I}_{j_{n}}$ we have

$$
E_{n} 1_{I}=\frac{|I|}{|A(I)|} 1_{A(I)} .
$$

Therefore by (3.29) and (3.30),

$$
\left|z_{I, \sigma(I)}\right| 1_{\sigma(I)} \leq\left|z_{I, \sigma(I)}\right| 1_{A(I)} \leq C_{\gamma} E_{n} 1_{I} .
$$

Let $\left\{r_{I}: I \in \mathcal{F}^{\prime}\right\}$ be an enumeration of the Rademacher system. Then by the UMD property, Kahane's contraction principle and E. M. Stein's martingale inequality we obtain

$$
\left\|\sum_{I \in \mathcal{F}^{\prime}} a_{I} Z\left(h_{I}\right)\right\|_{L^{p}(X)} \leq C_{p} E\left\|\sum_{I \in \mathcal{F}^{\prime}} a_{I} r_{I} z_{I, \sigma(I)} h_{\sigma(I)}\right\|_{L^{p}(X)} .
$$

Applying twice the contraction principle of Kahane gives these bounds for 
the right hand side of the above estimate:

$$
C_{p} E\left\|\sum_{I \in \mathcal{F}^{\prime}} a_{I} r_{I} z_{I, \sigma(I)} 1_{\sigma(I)}\right\|_{L^{p}(X)} \leq C_{p, \gamma} E\left\|\sum_{I \in \mathcal{F}^{\prime}} a_{I} r_{I} E_{n} 1_{I}\right\|_{L^{p}(X)} .
$$

By J. Bourgain's version of E. M. Stein's martingale inequality, the last expression is bounded as

$$
C_{p, \gamma} E\left\|\sum_{I \in \mathcal{F}^{\prime}} a_{I} r_{I} 1_{I}\right\|_{L^{p}(X)}
$$

Again the contraction principle of Kahane and the UMD property of $X$ imply that the last term is bounded by $\left\|\sum_{I \in \mathcal{F}^{\prime}} a_{I} h_{I}\right\|_{p}$. This gives (3.28) and (3.27). To deduce (3.1) note that the length of an interval $\Delta \in \mathcal{I}$ is comparable with the length of each of its "grandchildren". So when we start with $T$ where $x_{I, \tau(I)}$ satisfies (3.2), then the induced $z_{I, \sigma(I)}$ satisfies (3.30).

3.3. Boundedness of the splitting operator. In this section we prove the norm estimates for $U_{m}$. As in the treatment of $T_{m}$ the upper bound $\left\|U_{m}\right\|_{L^{p}(X)} \leq C \log (|m|+2)$ follows from an application of J. Bourgain's version of E. M. Stein's martingale inequality. Here however we stay much closer to Figiel's original proof in Section 6 of [6].

Let $U=U_{m}$ be defined by (3.3). We consider only the case $m>0$; the case $m<0$ is treated analogously. Let $\mathcal{F}$ be one of the collections $\mathcal{F}^{(r, s)}$ obtained from Proposition 3.4 with parameter $m$ and rearrangement $\tau_{m}$. We assume that $\mathcal{F}$ satisfies (3.17) and (3.18). The norm estimates for $U$ follow below by showing that $\mathcal{F}$ can be further decomposed so that its components either satisfy Figiel's compatibility condition or can be related to it. We now recall this notion.

3.3.1. Figiel's compatibility condition.. For $\mathcal{F}_{0} \subset \mathcal{F}$ we define

$$
\Omega\left(\mathcal{F}_{0}\right)=\left\{I, \tau(I): I \in \mathcal{F}_{0}\right\} .
$$

Let $\Delta \in \Omega\left(\mathcal{F}_{0}\right)$. Then we define $\Delta^{*}$ by the following rule:

$$
\Delta^{*}= \begin{cases}\tau(\Delta) & \text { if } \Delta \in \mathcal{F}_{0}, \\ \tau^{-1}(\Delta) & \text { if } \Delta \in \tau\left(\mathcal{F}_{0}\right) .\end{cases}
$$

We say that $\mathcal{F}_{0} \subset \mathcal{F}$ satisfies Figiel's compatibility condition if $I \subset J$ and $I \neq J$ implies that $I^{*} \subset J$, for any choice of $I, J \in \Omega\left(\mathcal{F}_{0}\right)$.

Recall that by (2.3) we imposed a canonical enumeration of the intervals of $\mathcal{I}$. Thus $\mathcal{I}=\left\{I_{n}: n \in \mathbb{N}\right\}$. Let $\left\{n_{k}\right\}$ be a subsequence such that $\mathcal{F}_{0}=$ $\left\{I_{n_{k}}: k \in \mathbb{N}\right\}$. With this enumeration, we introduce the following sequence of $\sigma$-algebras: 


$$
\begin{aligned}
\mathcal{T}_{1} & =\sigma\left(\left\{I_{n_{1}} \cup \tau\left(I_{n_{1}}\right)\right\}\right), \\
\mathcal{T}_{2 k} & =\sigma\left(\mathcal{T}_{2 k-1} \cup\left\{I_{n_{k}}\right\}\right), \quad k \geq 1, \\
\mathcal{T}_{2 k-1} & =\sigma\left(\mathcal{T}_{2 k-2} \cup\left\{I_{n_{k}} \cup \tau\left(I_{n_{k}}\right)\right\}\right), \quad k \geq 2 .
\end{aligned}
$$

Recall also that we put

$$
g_{I, \tau_{m}(I)}=1_{\tau_{m}(I)}-\frac{\left|\tau_{m}(I)\right|}{|I|} 1_{I}
$$

Since here we have already fixed $m$ we simplify our notation to

$$
g_{I}=g_{I, \tau_{m}(I)}
$$

The following fact is just a restatement of Figiel's compatibility condition.

FACT 3.11. Let $\mathcal{F}_{0} \subset \mathcal{F}$ and let $\left\{n_{k}\right\}$ be such that

$$
\mathcal{F}_{0}=\left\{I_{n_{k}}: k \in \mathbb{N}\right\} \text {. }
$$

Assume that $\mathcal{F}_{0}$ satisfies Figiel's compatibility condition. Then the sequence $\left\{g_{I}: I \in \mathcal{F}_{0}\right\}=\left\{g_{I_{n_{k}}}: k \in \mathbb{N}\right\}$ is a martingale difference sequence with respect to the $\sigma$-algebras $\mathcal{T}_{2 k}$.

The next proposition reveals the significance of Figiel's compatibility condition.

Proposition 3.12. Let $\mathcal{F}_{0} \subset \mathcal{F}$ satisfy Figiel's compatibility condition. Let $a_{I} \in X$. Then

$$
\left\|\sum_{I \in \mathcal{F}_{0}} a_{I} U h_{I}\right\|_{L^{p}(X)} \leq C\left\|\sum_{I \in \mathcal{F}_{0}} a_{I} h_{I}\right\|_{L^{p}(X)} .
$$

Proof. Both $\left\{g_{I}: I \in \mathcal{F}_{0}\right\}$ and $\left\{h_{I}: I \in \mathcal{F}_{0}\right\}$ are martingale differences (with respect to suitable $\sigma$-algebras). Let $I \in \mathcal{F}_{0}$. Hence there exists $n_{k} \in \mathbb{N}$ so that $I=I_{n_{k}}$. Let $E_{I}$ denote the conditional expectation with respect to the $\sigma$-algebra $\mathcal{T}_{2 k-1}$. Then we have first the inequalities

$$
C_{\gamma} \frac{|I|}{|I|+|\tau(I)|} 1_{I \cup \tau(I)} \leq E_{I}\left|h_{I}\right| \leq \frac{|I|}{|I|+|\tau(I)|} 1_{I \cup \tau(I)},
$$

which implies that

$$
\left|y_{I, \tau(I)} 1_{\tau(I)}\right| \leq\left|y_{I, \tau(I)} 1_{I \cup \tau(I)}\right| \leq C E_{I}\left|h_{I}\right| .
$$

Notice also that

$$
\left|y_{I, \tau(I)}\right| \frac{|\tau(I)|}{|I|} 1_{I} \leq C \frac{|I|}{|I|+|\tau(I)|} \cdot \frac{|\tau(I)|}{|I|} 1_{I} \leq C_{\gamma}\left|h_{I}\right| .
$$

Let $\left\{r_{I}\right\}$ denote an enumeration of the Rademacher system. Using the UMD property we get

$$
\left\|\sum_{I \in \mathcal{F}_{0}} a_{I} U h_{I}\right\|_{L^{p}(X)} \leq C E\left\|\sum_{I \in \mathcal{F}_{0}} r_{I} a_{I} y_{I, \tau(I)} g_{I}\right\|_{L^{p}(X)} .
$$


By the triangle inequality and the definition of $g_{I}$ the above is bounded by

$$
C E\left\|\sum_{I \in \mathcal{F}_{0}} r_{I} a_{I} y_{I, \tau(I)} 1_{\tau(I)}\right\|_{L^{p}(X)}+C E\left\|\sum_{I \in \mathcal{F}_{0}} r_{I} a_{I} y_{I, \tau(I)} \frac{|\tau(I)|}{|I|} 1_{I}\right\|_{L^{p}(X)} .
$$

Next use Kahane's contraction principle to further bound the above norms by

$$
C E\left\|\sum_{I \in \mathcal{F}_{0}} r_{I} a_{I} E_{I}\left|h_{I}\right|\right\|_{L^{p}(X)}+C_{\gamma} E\left\|\sum_{I \in \mathcal{F}_{0}} r_{I} a_{I}\left|h_{I}\right|\right\|_{L^{p}(X)} .
$$

By the contraction principle and the UMD property of $X$ the second term of the last line is a constant multiple of $\left\|\sum_{I \in \mathcal{F}_{0}} a_{I} h_{I}\right\|_{p}$. It remains to treat the first one. E. M. Stein's martingale inequality yields

$$
C E\left\|\sum_{I \in \mathcal{F}_{0}} r_{I} a_{I} E_{I}\left|h_{I}\right|\right\|_{L^{p}(X)} \leq C_{\gamma} E\left\|\sum_{I \in \mathcal{F}_{0}} r_{I} a_{I}\left|h_{I}\right|\right\|_{L^{p}(X)} .
$$

Combining the above remarks finishes the proof of the proposition.

3.3.2. Proof of Theorem 3.2. By the next proposition we are able to extract three disjoint subcollections of $\mathcal{F}$ each of which satisfies Figiel's compatibility condition. We do this in such a way that the operator $U$ restricted to the remaining collection is bounded independently of $m$. Clearly by Proposition 3.12 this implies that $U$ restricted to $\operatorname{span}\left\{h_{I}: I \in \mathcal{F}\right\}$ is bounded on $L^{p}(X)$ independently of $m$. Since $\mathcal{I}$ is split into at most $C \log (|m|+2)$ collections such as $\mathcal{F}$, verifying Proposition 3.13 below completes the proof of Theorem 3.2.

Proposition 3.13. There exists a decomposition of $\mathcal{F}$ as

$$
\mathcal{F}=\mathcal{E} \cup \mathcal{G}_{\text {even }} \cup \mathcal{G}_{\text {odd }} \cup \mathcal{L}
$$

so that

1. The collections $\mathcal{E}, \mathcal{G}_{\text {even }}, \mathcal{G}_{\text {odd }}$ satisfy Figiel's compatibility condition.

2. The operator $U$ restricted to $\operatorname{span}\left\{h_{I}: I \in \mathcal{L}\right\}$ is bounded on $L^{p}(X)$ (with norm estimates independent of $m$ ).

We obtain the proof of Proposition 3.13 by rewriting and rearranging Figiel's main combinatorial construction in [6, Section 6]. This is possible since Figiel's combinatorics exploits just the quasi-dyadic structure, and not any of the more special properties of dyadic intervals.

Proof. Let $\kappa=\kappa_{m}$ be as defined in the course of proving Proposition 3.4. For $I \in \mathcal{I}_{j}$ denote by $I^{(\kappa)}$ the unique interval from $\mathcal{I}_{j-\kappa}$ containing $I$. By the choice of $\kappa$, for each $I$, either $I^{(\kappa)}=(\tau(I))^{(\kappa)}$ or $\left(I^{(\kappa)}\right)^{+}=(\tau(I))^{(\kappa)}$. In the latter case, there are the following possibilities: none of $I, \tau(I)$ touches the common endpoint of $I^{(\kappa)}$ and $(\tau(I))^{(\kappa)}$, or exactly one of $I, \tau(I)$ touches the common endpoint of $I^{(\kappa)}$ and $(\tau(I))^{(\kappa)}$, or both $I, \tau(I)$ touch the common 
endpoint of $I^{(\kappa)}$ and $(\tau(I))^{(\kappa)}$ (this can happen only when $|m|=1$ ). Now, define

$$
\begin{aligned}
& \mathcal{E}=\left\{I \in \mathcal{F}: I^{(\kappa)}=(\tau(I))^{(\kappa)}\right\}, \\
& \mathcal{G}=\left\{I \in \mathcal{F}:\left(I^{(\kappa)}\right)^{+}=(\tau(I))^{(\kappa)} \text {, neither } I \text { nor } \tau(I)\right. \\
& \text { touch the common endpoint of } \left.I^{(\kappa)},(\tau(I))^{(\kappa)}\right\} \text {, } \\
& \mathcal{L}_{1,0}=\left\{I \in \mathcal{F}:\left(I^{(\kappa)}\right)^{+}=(\tau(I))^{(\kappa)} \text {, only } I\right. \\
& \text { touches the common endpoint of } \left.I^{(\kappa)},(\tau(I))^{(\kappa)}\right\} \text {, } \\
& \mathcal{L}_{0,1}=\left\{I \in \mathcal{F}: \quad\left(I^{(\kappa)}\right)^{+}=(\tau(I))^{(\kappa)} \text {, only } \tau(I)\right. \\
& \text { touches the common endpoint of } \left.I^{(\kappa)},(\tau(I))^{(\kappa)}\right\} \text {, } \\
& \mathcal{L}_{1,1}=\left\{I \in \mathcal{F}:\left(I^{(\kappa)}\right)^{+}=(\tau(I))^{(\kappa)} \text {, both } I \text { and } \tau(I)\right. \\
& \text { touch the common endpoint of } \left.I^{(\kappa)},(\tau(I))^{(\kappa)}\right\} \text {, } \\
& \mathcal{L}=\mathcal{L}_{1,0} \cup \mathcal{L}_{0,1} \cup \mathcal{L}_{1,1} .
\end{aligned}
$$

Observe that for $m>1$, the collection $\mathcal{L}_{1,1}$ is empty, while for $m=1$ the collections $\mathcal{G}, \mathcal{L}_{1,0}, \mathcal{L}_{0,1}$ are empty.

In the following paragraphs we analyze - one by one-the collections $\mathcal{E}, \mathcal{G}, \mathcal{L}$. The simplest is $\mathcal{E}$ since almost by definition it satisfies Figiel's compatibility condition. The collection $\mathcal{G}$ is treated by first splitting it into a sequence of generations that are not the usual ones. Indeed, we define maximality with respect to $I$ and $\tau(I)$. Finally, the collection $\mathcal{L}$ is treated by relating it to a family that satisfies conditions analogous to the ones that hold for $\mathcal{G}$.

Collection $\mathcal{E}$. The collection $\Omega(\mathcal{E})$ satisfies the compatibility condition. Indeed, let $I, J \in \Omega(\mathcal{E})$ with $I \subset J, I \neq J$. Because of the vertical splittings by $\kappa$ quasi-dyadic levels, this implies $I^{(\kappa)} \subset J$. Now, observe that $I^{*} \subset$ $\left(I^{*}\right)^{(\kappa)}=I^{(\kappa)} \subset J$.

Collection $\mathcal{G}$. The collection $\mathcal{G}$ splits into the union of two collections $\mathcal{G}_{\text {even }}, \mathcal{G}_{\text {odd }}$ such that both $\Omega\left(\mathcal{G}_{\text {even }}\right)$ and $\Omega\left(\mathcal{G}_{\text {odd }}\right)$ satisfy the compatibility condition. Now, we describe this splitting. In this description, we split $\Omega(\mathcal{G})$ into successive generations with respect to $\subset$.

STEP 0. Let $\mathcal{G}_{0}$ be the collection of $I \in \mathcal{G}$ such that both $I$ and $\tau(I)$ are maximal in $\Omega(\mathcal{G})$ (with respect to $\subset$ ). Then let $\mathcal{G}_{0}^{\prime}=\mathcal{G} \backslash \mathcal{G}_{0}$. Note that $\Omega\left(\mathcal{G}_{0}^{\prime}\right)=\Omega(\mathcal{G}) \backslash \Omega\left(\mathcal{G}_{0}\right)$.

STEP $n+1$. Suppose that $\mathcal{G}_{0}, \ldots, \mathcal{G}_{n}$ have been defined. Then we let $\mathcal{G}_{n+1}$ be the collection of $I \in \mathcal{G}_{n}^{\prime}$ such that both $I$ and $\tau(I)$ are maximal in $\Omega\left(\mathcal{G}_{n}^{\prime}\right)$. Then we let

$$
\mathcal{G}_{n+1}^{\prime}=\mathcal{G}_{n}^{\prime} \backslash \mathcal{G}_{n+1}=\mathcal{G} \backslash \bigcup_{l=0}^{n+1} \mathcal{G}_{l}
$$


Observe that

$$
\Omega\left(\mathcal{G}_{n+1}^{\prime}\right)=\Omega\left(\mathcal{G}_{n}^{\prime}\right) \backslash \Omega\left(\mathcal{G}_{n+1}\right)=\Omega(\mathcal{G}) \backslash \bigcup_{l=0}^{n+1} \Omega\left(\mathcal{G}_{l}\right) .
$$

Recall that by the definition of $\tau$, if $I \in \mathcal{I}_{j}$, then $\tau(I) \in \mathcal{I}_{j}$ as well. This implies that each interval $I \in \mathcal{I}_{j_{n}} \cap \mathcal{G}$ is in some $\mathcal{G}_{l}$ with $l \leq n$. Therefore

$$
\mathcal{G}=\bigcup_{n=0}^{\infty} \mathcal{G}_{n} \text { and } \Omega(\mathcal{G})=\bigcup_{n=0}^{\infty} \Omega\left(\mathcal{G}_{n}\right)
$$

Now, we let

$$
\begin{aligned}
\mathcal{G}_{\text {even }} & =\left\{I \in \mathcal{G}: I \in \mathcal{G}_{2 i}, i \geq 0\right\}, \\
\mathcal{G}_{\text {odd }} & =\left\{I \in \mathcal{G}: I \in \mathcal{G}_{2 i+1}, i \geq 0\right\} .
\end{aligned}
$$

We check that $\mathcal{G}_{\text {even }}, \mathcal{G}_{\text {odd }}$ satisfy the compatibility condition. The proof is split into Facts $3.14-3.16$ below.

FACT 3.14. Let $\Delta \in \Omega(\mathcal{G})$. Then there is no other $\Delta^{\prime} \in \Omega(\mathcal{G})$ having a common endpoint with $\Delta$.

Proof. Let $\alpha$ be an endpoint of an interval from $\Omega(\mathcal{G})$. Let $J$ be a maximal such interval. Clearly there exists a unique index $j_{n}$ so that $J \in \mathcal{I}_{j_{n}}$. It follows by condition (ii) for splittings of $\mathcal{I}_{j_{n}}$ that $J^{+}, J^{-} \notin \Omega(\mathcal{G})$ (recall that we assume $m \geq 2$ ). Therefore there is no interval in $\mathcal{I}_{j_{n}} \cap \Omega(\mathcal{G})$ other than $J$ which has $\alpha$ as its endpoint. Next, if $J^{\prime} \in \mathcal{I}_{j_{l}}$ with $l>n$, and $\alpha$ is an endpoint of $J^{\prime}$, then $\alpha$ is also an endpoint of $\left(J^{\prime}\right)^{(\kappa)}$. But this is impossible for $J^{\prime} \in \Omega(\mathcal{G})$ : this is the defining property of the collection $\mathcal{G}$.

FACT 3.15. Let $I \in \Omega(\mathcal{G})$. Let $\alpha_{I}$ be the common endpoint of $I^{(\kappa)}$ and $\left(I^{*}\right)^{(\kappa)}$. Suppose that $J \in \Omega(\mathcal{G})$ is such that $I \subset J, I \neq J$ and $I^{*} \not \subset J$. Then $\alpha_{I}$ is an endpoint of $J$.

Proof. Before we give a detailed proof we point out that the conclusion is indeed obvious (draw the picture). Let $n, l$ be such that $\alpha_{I}=t_{j, 2 k-1}$ with $j_{n}<j \leq j_{n+1}$ and $I \in \mathcal{I}_{j_{l}}$. Then $l>n+1$. There are two cases to consider: 1) $J \in \mathcal{I}_{z}$ with $j \leq z \leq j_{l-1}$ and 2) $z \leq j_{n}$.

If $J \in \mathcal{I}_{z}$ with $j \leq z \leq j_{l-1}$ and $\alpha_{I}$ is not an endpoint of $J$, then $J$ is disjoint from both $I^{(\kappa)}$ and $\left(I^{*}\right)^{(\kappa)}$. Consequently, $J$ contains neither $I$ nor $I^{*}$.

If $z \leq j_{n}$, then $\alpha_{I}$ is not a point from $\mathcal{P}_{z}$. Consequently, if $J \in \mathcal{I}_{z}$, then either $J$ contains both $I^{(\kappa)}$ and $\left(I^{*}\right)^{(\kappa)}$ (so also both $I$ and $I^{*}$ ), or it is disjoint from both $I^{(\kappa)}$ and $\left(I^{*}\right)^{(\kappa)}$ (in this case, $J$ contains neither $I$ nor $I^{*}$ ).

FACT 3.16. Let $I, J \in \Omega(\mathcal{G})$, and let $\alpha_{I}$ be the common endpoint of $I^{(\kappa)}$ and $\left(I^{*}\right)^{(\kappa)}$. If $\alpha_{I}$ is an endpoint of $J$ and $J \in \Omega\left(\mathcal{G}_{i}\right)$, then $I \in \Omega\left(\mathcal{G}_{i+1}\right)$. 
Proof. Recall first the conclusion of Fact 3.15. If $\alpha_{I}$ is an endpoint of $J$, then one of $I^{(\kappa)},\left(I^{*}\right)^{(\kappa)}$ is included in $J$, and consequently one of $I, I^{*}$ is a proper subset of $J$. Therefore, $I, I^{*}$ cannot both be maximal intervals in $\Omega\left(\mathcal{G}_{l}^{\prime}\right)$ for $l \leq i-1$.

Let $\Delta \neq J$ be an interval from $\Omega(\mathcal{G})$ containing one of $I, I^{*}$ as a proper subset. Then it must contain either $I^{(\kappa)}$ or $\left(I^{*}\right)^{(\kappa)}$; in any case, $\alpha_{I} \in \Delta$. By Fact 3.14, $\alpha_{I}$ is not an endpoint of $\Delta$. This implies that $\alpha_{I} \notin \mathcal{P}_{z}$, where $z$ is such that $\Delta \in \mathcal{I}_{z}$. As $\alpha_{I}$ is an endpoint of $J$, it follows that $J \subset \Delta$, and $J$ is a proper subset of $\Delta$. Since $J$ is a maximal interval in $\Omega\left(\mathcal{G}_{i-1}^{\prime}\right)$, the last inclusion implies that $\Delta \notin \Omega\left(\mathcal{G}_{i-1}^{\prime}\right)$, and consequently $\Delta \in \Omega\left(\mathcal{G}_{l}\right)$ for some $l<i$.

The above considerations imply that both $I$ and $I^{*}$ are maximal intervals in $\Omega\left(\mathcal{G}_{i}^{\prime}\right)$. But this means that one of $I, I^{*}$ is in $\mathcal{G}_{i+1}$, and both are in $\Omega\left(\mathcal{G}_{i+1}\right)$.

The compatibility condition for $\mathcal{G}_{\text {even }}, \mathcal{G}_{\text {odd }}$ is now a consequence of Facts $3.14-3.16$.

Collection $\mathcal{L}_{1,0}$. Note that for $I \in \mathcal{L}_{1,0}, I^{-}$is the "dyadic brother" of $I$ in the sense that for $I \in \mathcal{L}_{1,0}$ the intervals $I, I^{-}$have a common dyadic predecessor within $\mathcal{I}$. Observe further that $\tau_{m}(I)=\tau_{m+1}\left(I^{-}\right)$, and if $y_{I, \tau_{m}(I)}$ satisfies (3.3) for $\tau_{m}$, then $y_{I^{-}, \tau_{m+1}\left(I^{-}\right)}=y_{I, \tau_{m}(I)}$ satisfies (3.4) for $\tau_{m+1}$, with the implied constant depending only on $C$ from (3.4) and $\gamma$.

Observe that $\left\{I^{-}: I \in \mathcal{L}_{1,0}\right\}$ is a subset of the collection of type $\mathcal{G}$ for $\tau_{m+1}$. By weak regularity, the linear extension of the mapping

$$
h_{I} \mapsto h_{I^{-}}, \quad I \in \mathcal{L}_{1,0},
$$

is bounded on $L^{p}(X)$. Therefore the linear extension of the mapping

$$
h_{I} \mapsto y_{I, \tau_{m}(I)} g_{I^{-}, \tau_{m+1}\left(I^{-}\right)}, \quad I \in \mathcal{L}_{1,0},
$$

is bounded on $L^{p}(X)$. Therefore, it remains to check the boundedness of

$$
h_{I} \rightarrow y_{I, \tau_{m}(I)} \cdot\left(g_{I, \tau_{m}(I)}-g_{I^{-}, \tau_{m+1}\left(I^{-}\right)}\right) \quad \text { for } I \in \mathcal{L}_{1,0} .
$$

But since $\tau_{m}(I)=\tau_{m+1}\left(I^{-}\right)$, we have

$$
\begin{aligned}
g_{I, \tau_{m}(I)}-g_{I^{-}, \tau_{m+1}\left(I^{-}\right)}= & \left(1_{\tau_{m}(I)}-\frac{\left|\tau_{m}(I)\right|}{|I|} 1_{I}\right) \\
& -\left(1_{\tau_{m+1}\left(I^{-}\right)}-\frac{\left|\tau_{m+1}\left(I^{-}\right)\right|}{\left|I^{-}\right|} 1_{I^{-}}\right) \\
= & \frac{\left|\tau_{m}(I)\right|}{|I|}\left(\frac{|I|}{\left|I^{-}\right|} 1_{I^{-}}-1_{I}\right) \\
= & \frac{\left|\tau_{m}(I)\right|}{|I|} c_{I, I^{-}} h_{I^{-} \cup I} \quad \text { with } \quad\left|c_{I, I^{-}}\right| \sim_{\gamma} \text { const. }
\end{aligned}
$$

It follows by condition (3.4) that 


$$
\left|y_{I, \tau_{m}(I)}\right| \cdot \frac{\left|\tau_{m}(I)\right|}{|I|} \cdot\left|c_{I, I^{-}}\right| \leq C_{\gamma} .
$$

Hence (3.31) is bounded since we have just showed that it is dominated by the rearrangement operator

$$
h_{I} \mapsto C_{\gamma} h_{I^{-} \cup I}, \quad I \in \mathcal{L}_{1,0} .
$$

Again by weak regularity of the partition the above rearrangement operator is $L^{p}(X)$ bounded.

Collection $\mathcal{L}_{0,1}$. Note that $\left(\tau_{m}(I)\right)^{+}=\tau_{m+1}(I)$, which implies that $\mathcal{L}_{0,1}$ is a subset of a collection of type $\mathcal{G}$ for $\tau_{m+1}$. For $I \in \mathcal{L}_{0,1}, \tau_{m}(I)$ and $\tau_{m+1}(I)$ are "dyadic brothers". Therefore, $y_{I, \tau_{m+1}(I)}=\frac{\left|\tau_{m}(I)\right|}{\left|\tau_{m+1}(I)\right|} y_{I, \tau_{m}(I)}$ satisfies (3.3), and consequently

$$
h_{I} \mapsto \frac{\left|\tau_{m}(I)\right|}{\left|\tau_{m+1}(I)\right|} y_{I, \tau_{m}(I)} g_{I, \tau_{m+1}(I)} \quad \text { for } I \in \mathcal{L}_{0,1}
$$

is bounded on $L^{p}(X)$. It remains to treat

$$
h_{I} \mapsto y_{I, \tau_{m}(I)}\left(g_{I, \tau_{m}(I)}-\frac{\left|\tau_{m}(I)\right|}{\left|\tau_{m+1}(I)\right|} g_{I, \tau_{m+1}(I)}\right) \quad \text { for } I \in \mathcal{L}_{0,1} .
$$

Applying just the definition of $g_{I, \tau(I)}(\mathrm{cf} .(3.3))$ we find that

$$
\begin{aligned}
g_{I, \tau_{m}(I)}-\frac{\left|\tau_{m}(I)\right|}{\left|\tau_{m+1}(I)\right|} g_{I, \tau_{m+1}} & =1_{\tau_{m}(I)}-\frac{\left|\tau_{m}(I)\right|}{\left|\tau_{m+1}(I)\right|} 1_{\tau_{m+1}(I)} \\
& =c_{\tau_{m}(I), \tau_{m+1}(I)} h_{\tau_{m}(I) \cup \tau_{m+1}(I)},
\end{aligned}
$$

with $\left|c_{\tau_{m}(I), \tau_{m+1}(I)}\right| \sim_{\gamma}$ const. Thus we reduced the boundedness of (3.32) to that of the rearrangement operator

$$
h_{I} \mapsto c_{\gamma} h_{\tau_{m}(I) \cup \tau_{m+1}(I)}, \quad I \in \mathcal{L}_{0,1} .
$$

Now, observe that $\mathcal{L}_{0,1}$ can be treated as a subset of one of the collections $\mathcal{F}$ considered for boundedness of $T_{m}$ or $T_{m+1}$. The estimate (3.3) for $y$.,'s implies the estimate (3.2) for the implied $x_{., .}$Therefore the mapping (3.32) can be obtained as a composition of suitable $T_{m}$ (or $T_{m+1}$ ) and a mapping of type $\varrho_{2}$, but considered for "children" of $\Delta$ instead of "grandchildren" (that is, "moving the Haar function one level up"). This implies the boundedness of $(3.32)$.

Collection $\mathcal{L}_{1,1}$. This collection, which appears only when $m=1$, is treated by combining the methods used for $\mathcal{L}_{1,0}$ and $\mathcal{L}_{0,1}$.

\section{References}

[1] S. V. Bochkarev, Some inequalities for the Franklin series, Anal. Math. 1 (1975), 249-257. 
[2] J. Bourgain, Vector valued singular integrals and the $H^{1}$-BMO duality, in: Israel Seminar on Geometrical Aspects of Functional Analysis (1983/84), XVI, Tel Aviv Univ., Tel Aviv, 1984, 23 pp.

[3] D. L. Burkholder, Martingales and singular integrals in Banach spaces, in: Handbook of the Geometry of Banach Spaces, Vol. I, W. B. Johnson and J. Lindenstraus (eds.), Elsevier, 2001, 233-269.

[4] Z. Ciesielski, Properties of the orthonormal Franklin system, Studia Math. 23 (1963), 141-157.

[5] Z. Ciesielski, P. Simon and P. Sjölin, Equivalence of Haar and Franklin bases in $L_{p}$ spaces, ibid. 60 (1977), 195-210.

[6] T. Figiel, On equivalence of some bases to the Haar system in spaces of vector-valued functions, Bull. Polish Acad. Sci. Math. 36 (1988), 409-411.

[7] -, Singular integral operators: a martingale approach, in: Geometry of Banach Spaces (Strobl, 1989), London Math. Soc. Lecture Note Ser. 158, Cambridge Univ. Press, Cambridge, 1990, 95-110.

[8] T. Figiel and P. Wojtaszczyk, Special bases in function spaces, in: Handbook of the Geometry of Banach Spaces, Vol. I, W. B. Johnson and J. Lindenstraus (eds.), Elsevier, 2001, 561-597.

[9] A. M. Garsia, Martingale Inequalities: Seminar Notes on Recent Progress, W. A. Benjamin, Reading, MA, 1973.

[10] G. G. Gevorkyan and A. Kamont, On general Franklin systems, Dissertationes Math. (Rozprawy Mat.) 374 (1998).

[11] - - - Unconditionality of general Franklin systems in $L^{p}[0,1], 1<p<\infty$, Studia Math. 164 (2004), 161-204.

[12] G. G. Gevorkyan and A. A. Sahakian, Unconditional basis property of general Franklin systems, Izv. Nats. Akad. Nauk Armenii Mat. 35 (2000), no 4, 7-25 (in Russian); English transl.: J. Contemp. Math. Anal. 35 (2000), no. 4, 2-22.

[13] P. F. X. Müller, Isomorphisms between $H^{1}$ Spaces, Monografie Mat. 66, Birkhäuser, Basel, 2005.

[14] P. F. X. Müller and G. Schechtman, Several results concerning unconditionality in vector valued $L^{p}$ and $H^{1}$ spaces, Illinois J. Math. 35 (1991), 220-233.

Institute of Mathematics

Polish Academy of Sciences

Abrahama 18

81-825 Sopot, Poland

E-mail: a.kamont@impan.gda.pl
Department of Mathematics

J. Kepler University Linz

A-4040 Linz, Austria

E-mail: pfxm@bayou.uni-linz.ac.at

Received October 5, 2005

Revised version August 2, 2006 